\title{
Genetic diversity and relatedness of Fasciola spp. isolates from different hosts and geographic regions revealed by analysis of mitochondrial DNA sequences
}

\author{
L. Ai ${ }^{\text {a,b,c,d,1 }}$, Y.B. Weng ${ }^{\text {c,1 }}$, H.M. Elsheikha ${ }^{e}$, G.H. Zhao ${ }^{\text {f }}$, S. Alasaad ${ }^{\mathrm{g}}$, J.X. Chen ${ }^{\mathrm{d}}$, J. Li ${ }^{\mathrm{c}}$, \\ H.L. Li $^{\mathrm{c}}$, C.R. Wang ${ }^{\mathrm{a}}$, M.X. Chen ${ }^{\mathrm{d}}$, R.Q. Lin ${ }^{\mathrm{b}, \mathrm{c}, *}$, X.Q. Zhu ${ }^{\mathrm{a}, \mathrm{b}, * *}$ \\ a College of Animal Science and Veterinary Medicine, Heilongjiang Bayi Agricultural University, Daqing, Heilongjiang Province 163319, PR China \\ b State Key Laboratory of Veterinary Etiological Biology, Key Laboratory of Veterinary Parasitology of Gansu Province, Lanzhou Veterinary Research Institute, \\ CAAS, Lanzhou, Gansu Province 730046, PR China \\ c College of Veterinary Medicine, South China Agricultural University, Guangzhou, Guangdong Province 510642, PR China \\ d National Institute of Parasitic Diseases, Chinese Center for Disease Control and Prevention, 207 Second Ruijin Road, Shanghai 200025, PR China \\ e School of Veterinary Medicine and Science, University of Nottingham, Sutton Bonington Campus, Loughborough LE12 5RD, UK \\ ${ }^{f}$ College of Veterinary Medicine, Northwest A \& F University, Yangling, Shaanxi Province 712100, PR China \\ ${ }^{g}$ Estación Biológica de Doñana, Consejo Superior de Investigaciones Científicas, Avda Americo Vespucio s/n, Seville 41092, Spain
}

Keywords:

Mitochondrial DNA (mtDNA)

Cytochrome c oxidase subunit 1 (cox1)

NADH dehydrogenase subunits 4 (nad4)

NADH dehydrogenase subunits 5 (nad5)

Phylogenetic analysis

Fasciola hepatica

Fasciola gigantica

The intermediate Fasciola

\begin{abstract}
a
The present study examined sequence variability in a portion of the mitochondrial cytochrome c oxidase subunit 1 (pcox1) and NADH dehydrogenase subunits 4 and 5 (pnad4 and pnad5) among 39 isolates of Fasciola spp., from different hosts from China, Niger, France, the United States of America, and Spain; and their phylogenetic relationships were re-constructed. Intra-species sequence variations were $0.0-1.1 \%$ for pcox $1,0.0-2.7 \%$ for pnad4, and $0.0-3.3 \%$ for pnad5 for Fasciola hepatica; $0.0-1.8 \%$ for pcox $1,0.0-2.5 \%$ for pnad4, and $0.0-4.2 \%$ for pnad5 for Fasciola gigantica, and $0.0-0.9 \%$ for pcox $1,0.0-0.2 \%$ for pnad4, and $0.0-1.1 \%$ for pnad5 for the intermediate Fasciola form. Whereas, nucleotide differences were $2.1-2.7 \%$ for pcox1, 3.1-3.3\% for pnad4, and $4.2-4.8 \%$ for pnad5 between F. hepatica and F. gigantica; were $1.3-1.5 \%$ for pcox $1,2.1-2.9 \%$ for pnad $4,3.1-3.4 \%$ for pnad5 between F. hepatica and the intermediate form; and were $0.9-1.1 \%$ for pcox $1,1.4-1.8 \%$ for pnad4, 2.2-2.4\% for pnad5 between F. gigantica and the intermediate form. Phylogenetic analysis based on the combined sequences of pcox1, pnad4 and pnad5 revealed distinct groupings of isolates of F. hepatica, F. gigantica, or the intermediate Fasciola form irrespective of their origin, demonstrating the usefulness of the mtDNA sequences for the delineation of Fasciola species, and reinforcing the genetic evidence for the existence of the intermediate Fasciola form.
\end{abstract}

\section{Introduction}

* Corresponding author at: State Key Laboratory of Veterinary Etiological Biology, Key Laboratory of Veterinary Parasitology of Gansu Province, Lanzhou Veterinary Research Institute, CAAS, Lanzhou, Gansu Province 730046, PR China. Tel.: +86 931 8342837; fax: +86931 8340977.

** Corresponding author at: College of Animal Science and Veterinary Medicine, Heilongjiang Bayi Agricultural University, Daqing, Heilongjiang Province 163319, PR China. Tel.: +86 459 6819197; fax: +86 4596819190.

E-mail addresses: rqlin@scau.edu.cn (R.Q. Lin),

xingquanzhu1@hotmail.com,xingquanzh@scau.edu.cn (X.Q. Zhu).
Fascioliasis is recognized as an important disease of domestic animals and humans worldwide, causing significant economic losses and public health concern (Spithill and Dalton, 1998; Mas-Coma et al., 2001, 2005). The tax-

\footnotetext{
1 These two authors contributed equally to this work.
} 
Table 1

Adult samples of Fasciola spp. used in the present study, as well as their GenBankaccession numbers for sequences of partial mitochondrial cytochrome c oxidase subunit 1 gene (pcox1), NADH dehydrogenase subunits 4 and 5 genes (pnad4 and pnad5).

\begin{tabular}{|c|c|c|c|c|c|c|}
\hline \multirow[t]{2}{*}{ Sample codes } & \multirow[t]{2}{*}{ Geographic origin } & \multirow[t]{2}{*}{ Host } & \multirow[t]{2}{*}{ Identity $^{\mathrm{a}}$} & \multicolumn{3}{|c|}{ GenBank accession number } \\
\hline & & & & $\operatorname{pcox} 1$ & pnad4 & pnad5 \\
\hline FgGXB26-29 & Guangxi, China & Buffalo & F. gigantica & GU112472-75 & GU121036-39 & GU121067-70 \\
\hline FhFG3, 6, 10, 12 & France & Goat & F. hepatica & GU112476-79 & GU121032-35 & GU121071-74 \\
\hline FhFG5, 8 & France & Goat & F. hepatica & GU112480-81 & GU121043-44 & GU121078-79 \\
\hline FhHLJC10-13 & Heilongjiang, China & Cattle & Intermediate form & GU112487-90 & GU121028-31 & GU121063-66 \\
\hline FgCAY1 & Ayorou, Niger & Cattle & F. gigantica & GU112458 & GU121047 & GU121086 \\
\hline FgCGA9 & Gaya, Niger & Cattle & F. gigantica & GU112459 & GU121048 & GU121092 \\
\hline FgCM1 & Malgorou-Gaya, Niger & Cattle & F.gigantica & FJ469983 & GU121049 & GU220573 \\
\hline FgSTO1 & Torodi, Niger & Sheep & F. gigantica & GU112460 & GU121050 & GU121087 \\
\hline FgCBE1 & Benin, Niger & Cattle & F. gigantica & GU112461 & GU121051 & GU121082 \\
\hline FgCMA1 & Maradi, Niger & Cattle & F. gigantica & GU112462 & GU121052 & GU121089 \\
\hline FgCTE1 & Tera, Niger & Cattle & F. gigantica & GU112463 & GU121053 & GU121091 \\
\hline FgCMA3 & Maradi, Niger & Cattle & F. gigantica & GU112464 & GU121054 & GU121090 \\
\hline FgSTE1 & Tera, Niger & Sheep & F. gigantica & GU112465 & GU121055 & GU121084 \\
\hline FgSTE2 & Tera, Niger & Sheep & F. gigantica & GU112468 & GU121058 & GU121083 \\
\hline FgCTO1 & Torodi, Niger & Cattle & F. gigantica & GU112466 & GU121056 & GU121085 \\
\hline FgSAY1 & Ayorou, Niger & Sheep & F. gigantica & GU112467 & GU121057 & GU121088 \\
\hline FhCTO6 & Torodi, Niger & Cattle & F. hepatica & GU112469 & GU121059 & GU121095 \\
\hline FhCTO12 & Torodi, Niger & Cattle & F. hepatica & FJ469984 & GU121060 & GU121096 \\
\hline FhCMA3 & Maradi, Niger & Cattle & F. hepatica & GU112470 & GU121061 & GU121093 \\
\hline FhCTE5 & Tera, Niger & Sheep & F. hepatica & GU112471 & GU121062 & GU121094 \\
\hline FhAM1, FhAM2 & USA & Cattle & F. hepatica & GU112482-83 & GU121045-46 & GU121080-81 \\
\hline FhGSG17-19 & Gansu, China & Goat & F. hepatica & GU112484-86 & GU121040-42 & GU121075-77 \\
\hline Fh2.3, Fh2.5 & Mallorca, Spain & Cattle & F. hepatica & GU112454-55 & GU121024-25 & GU220569-70 \\
\hline Fh23.3 & Bilbao, Spain & Sheep & F. hepatica & GU112457 & GU121027 & GU220571 \\
\hline Fh84.4 & Valencia, Spain & Horse & F. hepatica & GU112456 & GU121026 & GU220572 \\
\hline $\mathrm{FH}^{\mathrm{b}}$ & Australia & Unknown & F. hepatica & AF216697 & AF216697 & AF216697 \\
\hline
\end{tabular}

Adult samples of Fasciola spp. used in the present study, as well as their GenBank accession numbers for sequences of partial mitochondrial cytochrome c oxidase subunit 1 gene (pcox1), NADH dehydrogenase subunits 4 and 5 genes (pnad4 and pnad5).

a Identification of Fasciola to the species level was achieved by PCR as described by Ai et al. (2010a)

b FH represents the Australian F. hepatica with complete mitochondrial genome sequence available in GenBank under accession number AF216697.

onomic classification of the family Fasciolidae has been controversial for decades. Although several species have been described within the genus Fasciola, only Fasciola hepatica and Fasciola gigantica have been recognized taxonomically as the causative agents of fascioliasis in animals and humans (Yamaguti, 1958; Mas-Coma et al., 2005). Differentiation between F. hepatica and F. gigantica species is possible based on morphological characters, but it is difficult to accurately discern between isolates of the two species due to the inconsistency of morphological features (Kendall, 1965). Adding to this uncertainty is the presence of the poorly characterized intermediate Fasciola forms (Itagaki et al., 2009; Ichikawa and Itagaki, 2010).

The availability of molecular approaches has facilitated the identification and genetic characterization of morphologically similar parasites (Gasser, 1999). However, the search for reliable molecular markers suitable for lowlevel phylogenetic analysis remains a challenging problem. In addition, there is limited information concerning the genetic diversity in the natural populations of the genus Fasciola. The internal transcribed spacer (ITS) region of nuclear ribosomal DNA (rDNA) is the most widely used marker at this level to discriminate the predominately tropical F. gigantica from the temperate F. hepatica (Adlard et al., 1993; Huang et al., 2004; Ali et al., 2008; Ai et al., 2010a), and to identify the "intermediate Fasciola", which is thought to be hybrid/introgressed forms between F. hepatica and F. gigantica (Huang et al., 2004; Itagaki et al., 2005a,b, 2009; Ashrafi et al., 2006; Le et al., 2008). An

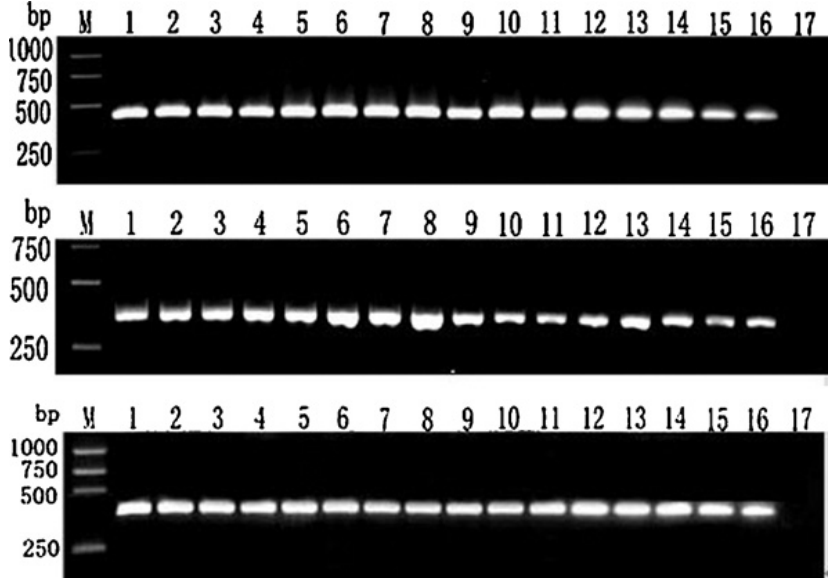

Fig. 1. Representative PCR products for a portion of the mitochondrial cytochrome c oxidase subunit 1 gene (pcox1, upper), NADH dehydrogenase subunit 4 gene (pnad4, middle) and subunit 5 gene (pnad5, bottom) of Fasciola isolates from China, Niger, France, USA and Spain. Lanes 1-17 represent samples FgGXB26, FgGXB27, FhFG3, FhFG10, FhHLJC10, FhHLJC11, FgCAY1, FgCBE1, FgCMA3, FhCTE5, FhCTO6, FhCMA3, FhFG5, FhFG8, FhAM1, FhAM2, and negative control, respectively. M represents a DNA size marker (ordinate values in bp).

earlier study by Semyenova et al. (2003) reported that individual cattle may be concurrently infected by more than one genotypes of the fluke based on random amplified polymorphic DNA (RAPD) genotyping analysis. In addition, the microsatellite markers have been employed to reveal the existence of genetic polymorphism between 
flukes from distinct definitive hosts (Hurtrez-Bousses et al., 2004).

Mitochondria are a valuable resource for studying the evolutionary process and deducing phylogeny. Recent studies showed that partial mitochondrial (mt) genes, such as a portion of cytochrome c oxidase subunit 1 (pcox1) and NADH dehydrogenase subunits 1 (pnad1) are considered to be good and useful markers to study the genetic differentiation and phylogenetic relationships among Fasciola species (Semyenova et al., 2006; Zarowiecki et al., 2007; Itagaki et al., 2009; Mera y Sierra et al., 2009).

The aims of the present study were to investigate Fasciola spp. isolates from different hosts and geographic locations in order to (1) determine the discriminatory potential of DNA sequence analysis based on three mitochondrial (mtDNA) regions, namely cox1, nad4 and nad5 for species differentiation, (2) examine intraspecies and interspecies genetic diversity, (3) clarify the taxonomic uncertainties of the "intermediate Fasciola form", and (4) to test hypotheses on Fasciola species monophyly.

\section{Materials and methods}

\subsection{Fasciola samples}

Thirty-nine Fasciola samples were collected from different geographical locations in China, Niger, France, USA and Spain. Sample codes, hosts and GenBank accession numbers are listed in Table 1. The four intermediate Fasciola samples were collected from four different cattle from four herds in Heilongjiang Province, China. Collected flukes were stored in $70 \%$ molecular grade ethanol, and stored at $-20{ }^{\circ} \mathrm{C}$ until extraction of genomic DNA.

\subsection{DNA extraction, PCR protocols and sequencing}

Total genomic DNA was extracted from individual flukes by using SDS/proteinase $\mathrm{K}$ treatment, column-purified (Wizard ${ }^{\circledR}$ SV Genomic DNA Purification System, Promega) and eluted into $60 \mathbf{- 1 ~} \mathrm{H}_{2} \mathrm{O}$ according to the manufacturer's recommendations (Zhao et al., 2009; Ai et al., 2010a,b). Each of the Fasciola samples was assigned to F. hepatica, F. gigantica or the intermediate Fasciola based on specific amplification of the ITS-2 rDNA sequence (Ai et al., 2010a).

A portion of the cox1 gene (pcox1) was amplified with primers JB3 $\left(5^{t}\right.$-TTTTTTGGGCATCCTGAGGTTTAT- $\left.3^{\mathrm{t}}\right)$ and JB4.5 (5t-TAAAGAAAGAACATAATGAAAATG$3^{t}$ ) (Bowles et al., 1992), part of the nad4 gene (pnad4) with primers ALF and ALR, and part of the nad5 gene (pnad5) with primers nad5F and nad5R. The primers ALF (5t-AGATGTCTATCCTTCCTT$\left.3^{\mathrm{t}}\right)$, ALR $\left(5^{\mathrm{t}}\right.$-ACTACCACAATATGTGCC- $\left.3^{\mathrm{t}}\right)$, nad5F $\left(5^{\mathrm{t}}\right.$-GCTATGCGCGCTCCTACTCCTGTTA- ${ }^{\mathrm{t}}$ ) and nad5R $\left(5^{t}\right.$-CTAGAACCAGACTGCCTCATCAAAT- $\left.3^{t}\right)$ were designed according to the complete mitochondrial genome sequence of the Australian F. hepatica (GenBank accession number AF216697). One $\mathbf{i}$ of DNA template was amplified in a 25il reaction volume containing $2 \mathrm{mM}$ of $\mathrm{MgCl}_{2}, 2.5 \mathbf{i} \mathrm{M}$ of each primer, 2.5 il 10× rTaq buffer, $0.2 \mathrm{mM}$ of each dNTPs and $1.25 \mathrm{U}$ of rTaq DNA polymerase (TAKARA). Amplification was performed in a thermocycler (Biometra) under the following conditions: after an initial denaturation at $94{ }^{\circ} \mathrm{C}$ for $5 \mathrm{~min}$, then $94{ }^{\circ} \mathrm{C}$ for $30 \mathrm{~s}$ (denaturation); $55{ }^{\circ} \mathrm{C}$ (for pcox1) or $50{ }^{\circ} \mathrm{C}$ (for pnad4) or $60{ }^{\circ} \mathrm{C}$ (for pnad5) for $30 \mathrm{~s}$ (annealing); $72{ }^{\circ} \mathrm{C}$ for $30 \mathrm{~s}$ (extension) for 35 cycles, followed by a final extension at $72{ }^{\circ} \mathrm{C}$ for $5 \mathrm{~min}$. Control samples without genomic DNA and host genomic DNA were included in each amplification run, and in no case were amplicons detected in the controls. Each amplicon (3 il) was electrophoresed by $1.5 \%$ agarose gel to validate amplification efficiency.

Positive amplicons were purified and sequenced in both directions using an ABI 377 automated DNA sequencer (using BigDye Terminator Chemistry) employing the same primers used in the PCR. The pcox1, pnad4 and pnad5 sequences are available from DDBJ, EMBL, and GenBank under the accession numbers shown in Table 1.

\subsection{Sequences analysis and phylogeny}

Sequences of the three mitochondrial genes were separately aligned using the computer program Clustal X 1.83 (Thompson et al., 1997). Pairwise comparisons were conducted of the level of sequence differences (D) among and within Fasciola taxa using the formula $D=1-(M / L)$, where $M$ is the number of alignment positions at which the two sequences have a base in common, and $\mathrm{L}$ is the total number of alignment positions over which the two sequences are compared (Chilton et al., 1995).

Sequences for each gene were individually aligned, and then concatenated into single alignments. Saturation levels of the aligned sequences at the first, second and third codon positions were separately assessed by plotting the uncorrected p distance for transitions versus transversions between pairs of sequences. The result (not shown) revealed the absence of saturation in any codon position, allowing the use of the alignment of the first, second and third codons for phylogenetic re-construction. Three methods, namely neighbor joining (NJ), maximum likelihood (ML) and maximum parsimony (MP), were used for phylogenetic re-constructions. NJ and MP analysis were carried out using PAUP 4.0 Beta 10 programme (Swofford, 2002), and ML analyses were performed using PUZZLE 4.1 (Strimmer and von Haeseler, 1996) under the default setting. The consensus tree was obtained after bootstrap analysis, with 1000 replications, with values above 50\% reported. To study the genetic relatedness with other Fasciola spp. samples, F. hepatica (FH) (GenBank accession number AF216697), was included into the present study, with Schistosoma japonicum (SJ) (AF215860) and Ascaris suum (AS) (X54253.1) as the outgroups. Phylograms were drawn using the Tree View program version 1.65 (Page, 1996).

\section{Results and discussion}

3.1. Amplification and sequences of pcox1, pnad4 and pnad5

For each mtDNA region, no size variation was detected on agarose gel among any of the amplicons examined (Fig. 1). After trimming some base pairs at the begin- 


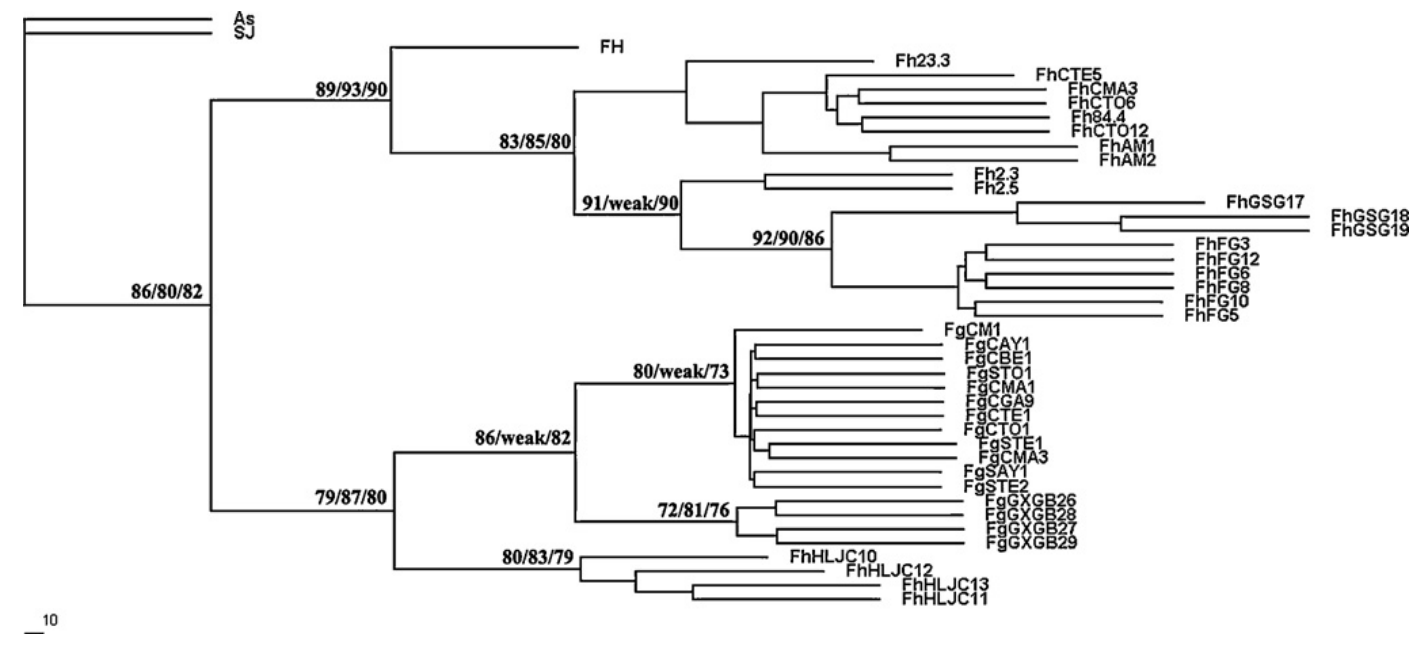

Fig. 2. Phylogenetic relationship of Fasciola spp. isolates from China, Niger, France, USA and Spain inferred by neighbor joining analysis using the combined dataset (cox1 + nad4 + nad5), with Schistosomajaponicum (SJ) and Ascaris suum (As) as the outgroups. Bootstrap values (in percentage) above 50\% from 1000 pseudo-replicates are shown for the neighbor-joining (the first value), maximum parsimony (the second value) and maximum likelihood analyses (the third value). Weak indicates nodes that are not well supported $(<50 \%)$. Scale bar indicates an evolutionary distance of 10 substitutions per site in the sequence. Refer to Table 1 for detail of Fasciola spp. isolates.

ning and end of the sequences, sequence size for pcox1 was $399 \mathrm{bp}, 463 \mathrm{bp}$ for pnad4 and $347 \mathrm{bp}$ for pnad5 for all of the examined Fasciola samples. The A+T contents of the sequences were 62.91-64.16\%(pcox1), 62.85-63.28\% (pnad4) and 62.54-63.11\% (pnad5).

\subsection{Sequence divergence}

The overall intraspecific nucleotide variations within F. hepatica were $0-1.1 \%$ for pcox $1,0-2.7 \%$ for pnad4, and $0-3.3 \%$ for pnad5. In F. gigantica, sequence variations were $0-1.8 \%$ for pcox $1,0-2.5 \%$ for pnad4, and $0-4.2 \%$ for pnad5. Within the intermediate Fasciola form, sequence variations were $0-0.9 \%$ for pcox $1,0-0.2 \%$ for pnad 4 , and $0-1.1 \%$ for pnad5. Whereas, interspecific sequence differences were higher than intraspecific nucleotide variations, being $2.1-2.7 \%$ for pcox $1,3.1-3.3 \%$ for pnad 4 and $4.2-4.8 \%$ for pnad5 between F. hepatica and F. gigantica; $1.3-1.5 \%$ for pcox1, 2.1-2.9\% for pnad4 and $3.1-3.4 \%$ for pnad5 between F. hepatica and the intermediate form; $0.9-1.1 \%$ for pcox 1 , $1.4-1.8 \%$ for pnad4 and $2.2-2.4 \%$ for pnad5 between $\mathrm{F}$. gigantica and the intermediate form. Nucleotide substitutions in sequences of the pcox1, pnad4 and pnad5 among Fasciola spp. isolates from different hosts and geographical locations were summarized in Electronic Supplementary Material.

Comparative analysis of different isolates of the same species from the same country exhibited small genetic variations for pcox1, pnad4 and pand5. For sequence differences in pcox1, samples from China were $0-0.1 \%$ for $\mathrm{F}$. hepatica isolates, and $0-0.1 \%$ for the intermediate form, and no sequence variation was detected for $F$. gigantica samples; samples from Niger were $0-0.2 \%$ for F. hepatica, and $0-0.3 \%$ for F. gigantica; F. hepatica samples from France and Spain were $0-0.1 \%$ and $0-0.3 \%$, respectively. For sequence variation in pnad4, samples from China were $0-0.2 \%$ for $\mathrm{F}$. hepatica, and equal for $\mathrm{F}$. gigantica samples and intermediate forms (0-0.1\%); samples from Niger were $0-0.1 \%$ for $\mathrm{F}$. hepatica, and $0-0.3 \%$ for F. gigantica; F. hepatica samples from France and Spain were $0-0.1 \%$ and $0-0.2 \%$, respectively. For sequence variations in pnad5, samples from China were $0-0.3 \%$ for $F$. hepatica, $0-0.2 \%$ for F. gigantica, but no genetic difference was found for intermediate forms; samples from Niger were $0-0.1 \%$ for F. hepatica, and $0-0.5 \%$ for F. gigantica; F. hepatica samples from France and Spain were $0-0.2 \%$ and $0-0.3 \%$, respectively. However, no sequence variation in the three mtDNA regions was detected between the two F. hepatica samples from USA.

For the pcox1, intraspecific nucleotide variation was related mainly to changes at the first and third codon positions in all of the three species, while no changes were detected at the second codon position. For pnad4 and pnad5, there were changes in the first, second and third codon positions. For the pnad4, there was only one change in the second codon of F. hepatica; but for pnad5, both F. hepatica and F. gigantica had one change in the second codon; and the variations in intermediate forms are mainly at the first and third codon positions.

\subsection{Phylogenetic relationships}

The combined sequences of pcox 1 , pnad4 and pnad5 were aligned over a consensus length of $1209 \mathrm{bp}$. Topologies of all trees inferred by different methods (NJ, MP, and ML) with different building strategies and/or different distance models were identical, with only small differences in bootstrap values (Fig. 2). The phylogenetic tree consisted of three large clades: F. hepatica, F. gigantica and the intermediate form. All the isolates of F. hepatica from different regions in different countries clustered together, supported by high bootstrap value ( $>50 \%)$. The isolates of $\mathrm{F}$. gigantica from China and Niger clustered together. The four intermediate Fasciola form isolates (FhHLJC10-13) from four cattle of different herds from Heilongjiang Province, China clustered together and exhibited more relatedness to F. gigantica than to $\mathrm{F}$. hepatica, reinforcing previous observation (Nguyen et al., 2009). 
Interestingly, we observed geographical isolation of $\mathrm{F}$. gigantica, where isolates of $\mathrm{F}$. gigantica from China and Niger were grouped in separate sub-clusters. However, no distinct geographical difference was observed for isolates of F. hepatica. Given that multiple mitochondrial lineages of F. hepatica have been documented within infrapopulations from cattle and sheep (Walker et al., 2007), further studies using more Fasciola samples, in particular more samples from a wider range of host species and broader geographic localities are needed for full clarification of the population genetic structures of Fasciola spp.

Taken together, our results indicate that phylogenetic analysis based on partial sequences of three mitochondrial genes (pcox1, pnad4 and pnad5) from 39 samples belonging to three Fasciola taxa supported the monophyly of the genus Fasciola and allowed better assessment of the genetic diversity and relatedness of various Fasciola species circulating in nature from different hosts and various geographic regions. Among the three mtDNA genes, sequence variation in pnad5 was higher compared to those of pcox1 and pnad4, and pnad 4 sequences exhibited more variability than the pcox1. This information is expected to yield new approaches for mitigating parasite transmission in animals and eventually for improving the control of fascioliasis.

\section{Conflict of interest statement}

The authors declare that they have no conflict of interests.

\section{Acknowledgements}

Project support was provided by grant from the Program for Changjiang Scholars and Innovative Research Team in University (Grant no. IRT0723), and the State Key Laboratory of Veterinary Etiological Biology, Lanzhou Veterinary Research Institute, Chinese Academy of Agricultural Sciences (SKLVEB2009KFKT014 and SKLVEB2010KFKT010). We thank Mr. Ali Halidou of Veterinary Laboratory, Ministry of Animal Resources, Niger for providing Fasciola samples from Niger. Also, we thank Prof. Sung-Jong Hong of the College of Medicine, Chung-Ang University, The Republic of Korea for providing Fasciola samples from the USA. Fasciola samples from Spain are kindly provided by Margarita Buades (Head of the Sección de Mataderos, Islas Baleares).

\section{Appendix A. Supplementary data}

Supplementary data associated with this article can be found, in the online version, at doi:10.1016/j.vetpar.2011. 03.057.

\section{References}

Adlard, R.D., Barker, S.C., Blair, D., Cribb, T.H., 1993. Comparison of the second internal transcribed spacer (ribosomal DNA) from populations and species of Fasciolidae (Digenea). Int. J. Parasitol. 23, 423-425.

Ai, L., Dong, S.J., Zhang, W.Y., Elsheikha, H.M., Mahmmod, Y.S., Lin, R.Q., Yuan, Z.G., Shi, Y.L., Huang, W.Y., Zhu, X.Q., 2010a. Specific PCR-based assays for the identification of Fasciola species: their development, evaluation and potential usefulness in prevalence surveys. Ann. Trop. Med. Parasitol. 104, 65-72.

Ai, L., Li, C., Elsheikha, H.M., Hong, S.J., Chen, J.X., Chen, S.H., Li, X., Cai, X.Q., Chen, M.X., Zhu, X.Q., 2010b. Rapid identification and differentiation of Fasciola hepatica and Fasciola gigantica by a loop-mediated isothermal amplification (LAMP) assay. Vet. Parasitol. 174, 228-233.

Ali, H., Ai, L., Song, H.Q., Ali, S., Lin, R.Q., Seyni, B., Issa, G., Zhu, X.Q., 2008. Genetic characterisation of Fasciola samples from different host species and geographical localities revealed the existence of F. hepatica and F. gigantica in Niger. Parasitol. Res. 102, 1021-1024.

Ashrafi, K., Valero, M.A., Panova, M., Periago, M.V., Massoud, J., Mas-Coma, S., 2006. Phenotypic analysis of adults of Fasciola hepatica, Fasciola gigantica and intermediate forms from the endemic region of Gilan. Iran. Parasitol. Int. 55, 249-260.

Bowles, J., Blair, D., McManus, D.P., 1992. Genetic variants within the genus Echinococcus identified by mitochondrial DNA sequencing. Mol. Biochem. Parasitol. 54, 165-173.

Chilton, N.B., Gasser, R.B., Beveridge, I., 1995. Differences in a ribosomal DNA sequence of morphologically indistinguishable species within the Hypodontus macropi complex (Nematoda: Strongyloidea). Int. J. Parasitol. 25, 647-651.

Gasser, R.B., 1999. PCR-based technology in veterinary parasitology. Vet. Parasitol. 84, 229-258.

Huang, W.Y., He, B., Wang, C.R., Zhu, X.Q., 2004. Characterisation of Fasciola species from Mainland China by ITS-2 ribosomal DNA sequence. Vet. Parasitol. 120, 75-83.

Hurtrez-Bousses, S., Durand, P., Jabbour-Zahab, R., Guegan, J.F., Meunier, C., Bargues, M.D., Mas-Coma, S., Renaud, F., 2004. Isolation and characterisation of microsatellite markers in the liver fluke (Fasciola hepatica). Mol. Ecol. Notes 4, 689-690.

Ichikawa, M., Itagaki, T., 2010. Discrimination of the ITS1 types of Fasciola spp. based on a PCR-RFLP method. Parasitol. Res. 106, 757-761.

Itagaki, T., Kikawa, M., Terasaki, K., Shibahara, T., Fukuda, K., 2005a. Molecular characterization of parthenogenic Fasciola sp. in Korea on the basis of DNA sequences of ribosomal ITS1 and mitochondrial NDI gene. J. Vet. Med. Sci. 67, 1115-1118.

Itagaki, T., Kikawa, M., Sakaguchi, K., Shimo, J., Terasaki, K., Shibahara, T., Fukuda, K., 2005b. Genetic characterization of parthenogenic Fasciola sp. in Japan on the basis of the sequences of ribosomal and mitochondrial DNA. Parasitology 131, 679-685.

Itagaki, T., Sakaguchi, K., Terasaki, K., Sasaki, O., Yoshihara, S., Van Dung, T., 2009. Occurrence of spermic diploid and aspermic triploid forms of Fasciola in Vietnam and their molecular characterization based on nuclear and mitochondrial DNA. Parasitol. Int. 58, 81-85.

Kendall, S.B., 1965. Relationships between the species of Fasciola and their molluscan hosts. Adv. Parasitol. 3, 59-98.

Le, T.H., De, N.V., Agatsuma, T., Thi Nguyen, T.G., Nguyen, Q.D., McManus, D.P., Blair, D., 2008. Human fascioliasis and the presence of hybrid/introgressed forms of Fasciola hepatica and Fasciola gigantica in Vietnam. Int. J. Parasitol. 38, 725-730.

Mas-Coma, S., Funatsu, I.R., Bargues, M.D., 2001. Fasciola hepatica and lymnaeid snails occurring at a very high altitude in South America. Parasitology 123, S115-S127.

Mas-Coma, S., Bargues, M.D., Valero, M.A., 2005. Fascioliasis and other plant-borne trematode zoonoses. Int. J. Parasitol. 35, 1255-1278.

Mera y Sierra, R., Artigas, P., Cuervo, P., Deis, E., Sidoti, L., Mas-Coma, S., Bargues, M.D., 2009. Fascioliasis transmission by Lymnaea neotropica confirmed by nuclear rDNA and mtDNA sequencing in Argentina. Vet. Parasitol. 166, 73-79.

Nguyen, T.G., Van De, N., Vercruysse, J., Dorny, P., Le, T.H., 2009. Genotypic characterization and species identification of Fasciola spp. with implications regarding the isolates infecting goats in Vietnam. Exp. Parasitol. 123, 354-361.

Page, R.D., 1996. TREEVIEW: an application to display phylogenetic trees on personal computers. Comput. Appl. Biosci. 12, 357-358.

Semyenova, S.K., Morozova, E.V., Chrisanfova, G.C., Asatrian, A.M., Movsessian, S.O., Ryskov, A.P., 2003. RAPD variability and genetic diversity in two populations of the liver fluke, Fasciola hepatica. Acta Parasitol. 48, 125-130.

Semyenova, S.K., Morozova, E.V., Chrisanfova, G.G., Gorokhov, V.V., Arkhipov, I.A., Moskvin, A.S., Movsessyan, S.O., Ryskov, A.P., 2006. Genetic differentiation in eastern European and western Asian populations of the liver fluke. Fasciola hepatica, as revealed by mitochondrial nad1 and cox1 genes. J. Parasitol. 92, 525-530.

Spithill, T.W., Dalton, J.P., 1998. Progress in development of liver fluke vaccines. Parasitol. Today 14, 224-228.

Strimmer, K., von Haeseler, A., 1996. Quartet puzzling: a quartet maximum likelihood method for reconstructing tree topologies. Mol. Biol. Evol. 13, 964-969. 
Swofford, D.L., 2002. PAUP*: Phylogenetic Analysis Using Parsimony (and other Methods). Sinauer Associates, Sunderland, MA.

Thompson, J.D., Gibson, T.J., Plewniak, F., Jeanmougin, F., Higgins, D.G., 1997. The Clustal X windows interface: flexible strategies for multiple sequence alignment aided by quality analysis tools. Nucl. Acids Res. 24, 4876-4882.

Walker, S.M., Prodöhl, P.A., Fletcher, H.L., Hanna, R.E., Kantzoura, V., Hoey, E.M., Trudgett, A., 2007. Evidence for multiple mitochondrial lineages of Fasciola hepatica (liver fluke) within infrapopulations from cattle and sheep. Parasitol. Res. 101, 117-125.
Yamaguti, S., 1958. Systema Helminthum, Vol. I. The Digenetic Trematodes of Vertebrates. Interscience Publishers, Inc., New York.

Zarowiecki, M.Z., Huyse, T., Littlewood, D.T., 2007. Making the most of mitochondrial genomes-markers for phylogeny, molecular ecology and barcodes in Schistosoma (Platyhelminthes: Digenea). Int. J. Parasitol. 37, 1401-1418.

Zhao, G.H., Mo, X.H., Zou, F.C., Li, J., Weng, Y.B., Lin, R.Q., Xia, C.M., Zhu, X.Q. 2009. Genetic variability among Schistosoma japonicum isolates from different endemic regions in China revealed by sequences of three mitochondrial DNA genes. Vet. Parasitol. 162, 67-74. 